\title{
Connecting orbits for a periodically forced singular planar Newtonian system
}

\author{
Marek Izydorek and Joanna Janczewska
}

To Prof. Kazimierz Gȩba on the occasion of his 80th birthday

\begin{abstract}
In this paper we are concerned with the study of the existence and multiplicity of connecting orbits for a singular planar Newtonian system $\ddot{q}+V_{q}(t, q)=0$ with a periodic strong force $V_{q}(t, q)$, an infinitely deep well of Gordon's type at one point and two stationary points at which a potential $V(t, q)$ achieves a strict global maximum. To this end we minimize the corresponding action functional over the classes of functions in the Sobolev space $W_{\text {loc }}^{1,2}\left(\mathbb{R}, \mathbb{R}^{2}\right)$ that turn a given number of times around the singularity.
\end{abstract}

Mathematics Subject Classification. Primary 34C37; Secondary 49M10.

Keywords. Heteroclinic orbits, homoclinic orbits, Newtonian systems.

\section{Introduction}

In this paper, we study the existence of connecting orbits for a certain class of planar singular Newtonian systems that are periodically forced. Let us consider a system

$$
\ddot{q}+V_{q}(t, q)=0, \quad q \in \mathbb{R}^{2},
$$

with a potential $V$ satisfying the following assumptions:

$(V 1) V \in C^{1}\left(\mathbb{R} \times\left(\mathbb{R}^{2} \backslash\{\xi\}\right), \mathbb{R}\right)$ is a $\tau$-periodic potential with respect to $t$, $(V 2) \lim _{x \rightarrow \xi} V(t, x)=-\infty$ uniformly in $t$,

$(V 3)$ there is a neighbourhood $\mathcal{N}$ of the point $\xi$ and there is a function $U \in$ $C^{1}(\mathcal{N} \backslash\{\xi\}, \mathbb{R})$ such that $|U(x)| \rightarrow \infty$ as $x \rightarrow \xi$ and for all $x \in \mathcal{N} \backslash\{\xi\}$ and $t \in \mathbb{R}$,

$$
|\nabla U(x)|^{2} \leq-V(t, x),
$$

(V4) $V(t, x) \leq 0$ and $V(t, x)=0$ if and only if $x \in \mathcal{M}$, where $\mathcal{M}$ is a set composed of two distinct points $a, b \in \mathbb{R}^{2} \backslash\{\xi\}$, 
(V5) there is a negative real constant $V_{0}$ such that for all $t \in \mathbb{R}$,

$$
\lim \sup V(t, x) \leq V_{0} \text {. }
$$

Without loss of generality, we can assume that $\tau=1$.

Definition 1.1. We say that $q: \mathbb{R} \rightarrow \mathbb{R}^{2} \backslash\{\xi\}$ is a connecting orbit of (1.1) if it is a solution of (1.1) that emanates from $\mathcal{M}$ and terminates at $\mathcal{M}$, i.e., $q( \pm \infty)=\lim _{t \rightarrow \pm \infty} q(t) \in \mathcal{M}$, and $\dot{q}( \pm \infty)=0$. Moreover, if $q(-\infty)=$ $q(\infty)$, then $q$ is called a homoclinic orbit, and if $q(-\infty) \neq q(\infty)$, then $q$ is a heteroclinic one. If $q(t) \equiv a(q(t) \equiv b)$ for all $t \in \mathbb{R}$, then $q$ is said to be a trivial orbit.

Let

$$
E=\left\{q \in W_{\mathrm{loc}}^{1,2}\left(\mathbb{R}, \mathbb{R}^{2}\right): \int_{-\infty}^{\infty}|\dot{q}(t)|^{2} d t<\infty\right\}
$$

be the Hilbert space equipped with the norm

$$
\|q\|=\left(\int_{-\infty}^{\infty}|\dot{q}(t)|^{2} d t+|q(0)|^{2}\right)^{\frac{1}{2}} .
$$

In this space we are interested in paths that omit the singularity $\xi$,

$$
\Lambda=\{q \in E: q(t) \neq \xi \text { for all } t \in \mathbb{R}\} .
$$

For $q \in \Lambda$, set

$$
I(q)=\int_{-\infty}^{\infty}\left(\frac{1}{2}|\dot{q}(t)|^{2}-V(t, q(t))\right) d t .
$$

From ( $V 3)$, the strong force condition due to Gordon [3], we immediately conclude that if $q \in \Lambda$ and $q(t) \in \mathcal{N}$ for all $t \in[r, s]$, then

$$
|U(q(s))| \leq|U(q(r))|+\left(\int_{r}^{s}-V(t, q(t)) d t\right)^{\frac{1}{2}} \cdot\left(\int_{r}^{s}|\dot{q}(t)|^{2} d t\right)^{\frac{1}{2}},
$$

and hence

$$
|U(q(s))| \leq|U(q(r))|+\sqrt{2} I(q),
$$

(compare [9, (2.21), p. 271]). In the literature, if a potential $V(t, q)$ satisfies the Gordon condition, then the gradient $V_{q}(t, q)$ is called a strong force. This condition governs the rate at which $V(t, q) \rightarrow-\infty$ as $q \rightarrow \xi$ and holds, for instance, if $\beta \geq 2$ for $V(q)=-|q-\xi|^{-\beta}$ nearby $\xi$.

We denote by $B_{\varepsilon}(x)$ a ball in $\mathbb{R}^{2}$ of radius $\varepsilon$, centered at a point $x \in \mathbb{R}^{2}$. Define

$$
R=\frac{1}{3} \min \{|b-a|,|\xi-a|,|\xi-b|\} .
$$

Lemma 1.2. Fix $0<\varepsilon \leq R$. If $q \in \Lambda$ and $q(t) \notin B_{\varepsilon}(\mathcal{M})$ for each $t \in$ $\bigcup_{i=1}^{k}\left[r_{i}, s_{i}\right]$, where $\left[r_{i}, s_{i}\right] \cap\left[r_{j}, s_{j}\right]=\emptyset$ for $i \neq j$, then

$$
I(q) \geq \sqrt{2 \alpha_{\varepsilon}} \sum_{i=1}^{k}\left|q\left(s_{i}\right)-q\left(r_{i}\right)\right|,
$$

where $\alpha_{\varepsilon}=\inf \left\{-V(t, x): t \in \mathbb{R}, x \notin B_{\varepsilon}(\mathcal{M})\right\}$ and $B_{\varepsilon}(\mathcal{M})=B_{\varepsilon}(a) \cup B_{\varepsilon}(b)$. 
The proof of Lemma 1.2 is the same as that of [8, Lemma 3.6] or [5, Lemma 2.1]. Applying Lemma 1.2 we obtain the following conclusion.

Corollary 1.3. If $q \in \Lambda$ and $I(q)<\infty$, then $q \in L^{\infty}\left(\mathbb{R}, \mathbb{R}^{2}\right)$ and $q( \pm \infty) \in \mathcal{M}$.

Lemma 1.4. Let $\left\{q_{m}\right\}_{m \in \mathbb{N}} \subset \Lambda$. If the value sequence $\left\{I\left(q_{m}\right)\right\}_{m \in \mathbb{N}}$ is bounded in $\mathbb{R}$, then there is $\delta>0$ such that $q_{m}(\mathbb{R}) \cap B_{\delta}(\xi)=\emptyset$ for all $m \in \mathbb{N}$.

The above lemma is a consequence of inequality (1.3) and Corollary 1.3.

In the polar coordinate system with the pole $\xi$ and the polar axis $\{x \in$ $\left.\mathbb{R}^{2}: x=\xi+s \cdot \overrightarrow{\xi a}, s \geq 0\right\}$ one has $q(t)=(r(t) \cos \varphi(t), r(t) \sin \varphi(t))$ for all $q \in \Lambda$ (polar angles are measured counterclockwise from the axis). Moreover, we can assume that $r(t)$ and $\varphi(t)$ are continuous.

Definition 1.5. For each $q \in \Lambda$ such that $q( \pm \infty) \in \mathcal{M}$, we define the rotation (winding) number $\operatorname{rot}(q)$ as follows. If $q(-\infty)=b$ and $q(\infty)=a$, then

$$
\operatorname{rot}(q)=\left[\frac{\varphi(\infty)-\varphi(-\infty)}{2 \pi}\right]+1
$$

Otherwise,

$$
\operatorname{rot}(q)=\left[\frac{\varphi(\infty)-\varphi(-\infty)}{2 \pi}\right] .
$$

Here $[s]$ denotes the integral part of $s \in \mathbb{R}$.

We denote by $\mathcal{F}$ the collection of all subsets $Z$ of $\Lambda$ that satisfy the following three conditions:

(i) if $q \in Z$, then $I(q)<\infty$,

(ii) if $p, q \in Z$, then $p( \pm \infty)=q( \pm \infty)$,

(iii) if $q \in Z$, then $q+s \psi \in Z$ for all $\psi \in C_{0}^{\infty}\left(\mathbb{R}, \mathbb{R}^{2}\right)$ and $s \in \mathbb{R}$ small enough.

Define

$$
\begin{aligned}
\Gamma^{+} & =\{q \in \Lambda: q(-\infty)=a, q(\infty)=b \wedge \operatorname{rot}(q) \geq 0\}, \\
\Gamma^{-} & =\{q \in \Lambda: q(-\infty)=a, q(\infty)=b \wedge \operatorname{rot}(q)<0\}, \\
\hat{\Omega}_{a}^{ \pm n} & =\{q \in \Lambda: q( \pm \infty)=a \wedge \pm \operatorname{rot}(q) \geq n\}, \\
\hat{\Omega}_{b}^{ \pm n} & =\{q \in \Lambda: q( \pm \infty)=b \wedge \pm \operatorname{rot}(q) \geq n\},
\end{aligned}
$$

where $n \in \mathbb{N}$. We see at once that these sets belong to $\mathcal{F}$.

Set

$$
\begin{gathered}
\gamma^{ \pm}=\inf \left\{I(q): q \in \Gamma^{ \pm}\right\}, \\
\hat{\omega}_{a}^{ \pm n}=\inf \left\{I(q): q \in \hat{\Omega}_{a}^{ \pm n}\right\}, \\
\hat{\omega}_{b}^{ \pm n}=\inf \left\{I(q): q \in \hat{\Omega}_{b}^{ \pm n}\right\} .
\end{gathered}
$$

From (iii) it follows that if $Z \in \mathcal{F}$ and $q$ is a minimizer of $I$ on $Z$, then $q$ is a weak solution of (1.1). Analysis similar to that in [8] (see the proof of Proposition 3.18, pp. 339-340) shows that $q$ is a classical solution. Moreover, in the same manner as in [4] (see the proof of Lemma 2.9, pp. 387-388) we can see that $\dot{q}( \pm \infty)=0$.

In the next section we will prove the following result. 
Theorem 1.6 (Main theorem). Suppose that $V$ satisfies $(V 1)-(V 5)$. Then (1.1) possesses at least two nontrivial connecting orbits.

There is always one heteroclinic solution of (1.1). The second solution may be heteroclinic or homoclinic depending on the geometry of $V$. In particular, there might be no homoclinics at all.

There are several papers on connecting orbits for a singular Newtonian system with a strong force via a variational approach. See for instance [1], $[2],[6],[7],[9],[10]$ and the references therein. The cited papers except [10] concern planar Newtonian systems.

Our work extends $[9$, Theorem 2.7] for the case $\# \mathcal{M}=1$ to the case $\# \mathcal{M}=2$ and $[7$, Conclusion 1.5] for an autonomous system to a periodic one. The proof of Theorem 1.6 is based on minimization of $I$ on appropriate sets in $\mathcal{F}$.

Connecting orbits are global in time. For this reason it is natural to apply global methods, in particular variational ones, to obtain them in a direct way, working with a class of functions possessing the qualitative properties sought.

The study of singular Hamiltonian systems seems to be important, because certain potentials arising in physics possess infinitely deep wells. As pointed out by Gordon in [3], it is a little disappointing that the strong force condition excludes gravitational potentials. However, he also wrote "the definition of the strong force condition is well motivated and leads to the disclosure of certain differences between the behaviour of strong force systems and gravitational (or other weak force) systems".

\section{Technicalities}

Before we show Theorem 1.6 we state and prove some technical results.

Proposition 2.1. Let $Z \in \mathcal{F}$. If $\left\{q_{m}\right\}_{m \in \mathbb{N}} \subset Z$ is a sequence such that the sequence of values $\left\{I\left(q_{m}\right)\right\}_{m \in \mathbb{N}}$ is bounded in $\mathbb{R}$, then $\left\{q_{m}\right\}_{m \in \mathbb{N}}$ is bounded in $E$.

Proof. We will apply the same arguments as in the proof of [7, Proposition 2.4]. By assumption, there exists a positive constant $M$ such that

$$
0<I\left(q_{m}\right) \leq M
$$

for each $m \in \mathbb{N}$. Hence

$$
\int_{-\infty}^{\infty}\left|\dot{q}_{m}(t)\right|^{2} d t \leq 2 M
$$

for each $m \in \mathbb{N}$. Fix $0<\varepsilon \leq R$. From Corollary 1.3 it follows that for each $m \in \mathbb{N}$ there are $r_{m}<s_{m}$ such that $q_{m}\left(\left(-\infty, r_{m}\right]\right) \cup q_{m}\left(\left[s_{m}, \infty\right)\right) \subset c l B_{\varepsilon}(\mathcal{M})$. Moreover, Lemma 1.2 implies the boundedness of the sequence

$$
\max _{t \in\left[r_{m}, s_{m}\right]}\left|q_{m}(t)\right|, \quad m \in \mathbb{N} .
$$


Therefore $\left\{q_{m}\right\}_{m \in \mathbb{N}}$ is bounded in $L^{\infty}\left(\mathbb{R}, \mathbb{R}^{2}\right)$. Consequently, $\left\{q_{m}\right\}_{m \in \mathbb{N}}$ is bounded in $E$.

From now on,

$$
\begin{aligned}
I_{T_{1}}^{T_{2}}(q) & =\int_{T_{1}}^{T_{2}}\left(\frac{1}{2}|\dot{q}(t)|^{2}-V(t, q(t))\right) d t, \\
I_{-\infty}^{T}(q) & =\int_{-\infty}^{T}\left(\frac{1}{2}|\dot{q}(t)|^{2}-V(t, q(t))\right) d t, \\
I_{T}^{\infty}(q) & =\int_{T}^{\infty}\left(\frac{1}{2}|\dot{q}(t)|^{2}-V(t, q(t))\right) d t
\end{aligned}
$$

for $T, T_{1}, T_{2} \in \mathbb{R}$ and $q \in \Lambda$.

Remark 2.2. It is easily seen that the functionals $I_{T_{1}}^{T_{2}}, I_{-\infty}^{T}, I_{T}^{\infty}$ for all $T_{1}, T_{2}, T \in \mathbb{R}$ and the functional $I$ are weakly lower semicontinuous.

Lemma 2.3. For each $\eta>0$ there exists $0<\delta \leq R$ such that if $x, y \in B_{\delta}(a)$ (or $x, y \in B_{\delta}(b)$ ) and $T \in \mathbb{R}$, then

$$
\int_{T}^{T+1}\left(\frac{1}{2}|y-x|^{2}-V\left(t, l_{x, y}(t)\right)\right) d t<\eta,
$$

where $l_{x, y}(t)=(T+1-t) x+(t-T)$ y for $t \in[T, T+1]$.

The proof is immediate and therefore it is left to the reader.

If $q \in \Lambda$ has the endpoints $q( \pm \infty)$ in $\mathcal{M}$, then for a time $T \in \mathbb{R}$ such that $q(T) \in B_{\varepsilon}(x)$, where $x \in \mathcal{M}$ and $\varepsilon>0$ small enough, we will denote by $\operatorname{rot}\left(\left.q\right|_{(-\infty, T]}\right)$ and $\operatorname{rot}\left(\left.q\right|_{[T, \infty)}\right)$ the rotation numbers of appropriate paths in $\Lambda$ that arise from $\left.q\right|_{(-\infty, T]}$ and $\left.q\right|_{[T, \infty)}$ resp. by connecting $q(T)$ to $x$ by a line segment.

Theorem 2.4. If $V$ satisfies $(V 1)-(V 5)$, then (1.1) possesses at least one heteroclinic orbit.

The proof is similar to that of [7, Theorem 1.3]. However, for the convenience of the reader we present it.

Proof. There is no loss of generality in assuming that

$$
\gamma^{-} \leq \gamma^{+}
$$

Let $\left\{q_{m}\right\}_{m \in \mathbb{N}} \subset \Gamma^{-}$be a minimizing sequence of $I$ in $\Gamma^{-}$, i.e.,

$$
\lim _{m \rightarrow \infty} I\left(q_{m}\right)=\gamma^{-} \text {. }
$$

Fix $0<\varepsilon \leq R$. For each $m \in \mathbb{N}$ there is $\tau_{m} \in \mathbb{R}$ such that $q_{m}\left(\tau_{m}\right) \in \partial B_{\varepsilon}(a)$ and if $t<\tau_{m}$, then $\left|q_{m}(t)-a\right|<\varepsilon$. Without loss of generality, we can assume that $\tau_{m} \in[0,1)$. By Proposition 2.1 it follows that there is $Q \in E$ such that going to a subsequence if necessary $q_{m}$ converges to $Q$ weakly in $E$ and strongly in $L_{\text {loc }}^{\infty}\left(\mathbb{R}, \mathbb{R}^{2}\right)$. From Remark 2.2 we obtain

$$
I(Q) \leq \gamma^{-},
$$


and by Corollary 1.3, $Q( \pm \infty) \in \mathcal{M}$. Lemma 1.4 gives $Q(t) \neq \xi$ for all $t \in \mathbb{R}$. Moreover, $Q((-\infty, 0]) \subset \operatorname{cl} B_{\varepsilon}(a)$, which gives $Q(-\infty)=a$. We will show now that $Q(\infty)=b$.

Conversely, suppose that $Q(\infty)=a$. Let $\delta>0$ be small enough such that $4 \delta<\varepsilon$

$$
2 \delta^{2}+\max \{-V(t, x): t \in \mathbb{R} \wedge|x-a| \leq 2 \delta\}<\frac{\varepsilon}{4} \sqrt{2 \alpha_{\frac{\varepsilon}{2}}}
$$

and there is $t_{\delta}>1$ such that $Q\left(t_{\delta}\right) \in \partial B_{\delta}(a)$ and $Q(t) \in B_{\delta}(a)$ for all $t>t_{\delta}$. Since $q_{m}\left(t_{\delta}\right) \rightarrow Q\left(t_{\delta}\right)$ as $m \rightarrow \infty$, there exists $m_{0} \in \mathbb{N}$ such that for all $m \geq m_{0}$,

$$
\left|q_{m}\left(t_{\delta}\right)-a\right|<2 \delta
$$

By the above, for each $m \geq m_{0}$ there is $s_{\delta}^{m} \in\left[\tau_{m}, t_{\delta}\right]$ such that if $t \in\left[\tau_{m}, s_{\delta}^{m}\right]$, then $q_{m}(t) \notin B_{\frac{\varepsilon}{2}}(a)$ and $q_{m}\left(s_{\delta}^{m}\right) \in \partial B_{\frac{\varepsilon}{2}}(a)$. Lemma 1.2 implies

$$
I\left(q_{m}\right) \geq \frac{\varepsilon}{2} \sqrt{2 \alpha_{\frac{\varepsilon}{2}}}+I_{t_{\delta}}^{\infty}\left(q_{m}\right) .
$$

Set

$$
Q_{m}(t)= \begin{cases}a & \text { if } t<t_{\delta}-1, \\ \left(t-t_{\delta}+1\right) q_{m}\left(t_{\delta}\right)+\left(t_{\delta}-t\right) a & \text { if } t \in\left[t_{\delta}-1, t_{\delta}\right], \\ q_{m}(t) & \text { if } t>t_{\delta},\end{cases}
$$

where $m \geq m_{0}$. We see that each $Q_{m}$ joins $a$ to $b$,

$$
I\left(Q_{m}\right)<I\left(q_{m}\right)-\frac{\varepsilon}{4} \sqrt{2 \alpha_{\frac{\varepsilon}{2}}},
$$

and consequently,

$$
\gamma^{-} \leq \liminf _{m \rightarrow \infty} I\left(Q_{m}\right) \leq \gamma^{-}-\frac{\varepsilon}{4} \sqrt{2 \alpha_{\frac{\varepsilon}{2}}}
$$

a contradiction. Therefore $Q(\infty)=b$.

To finish the proof we have to show that $\operatorname{rot}(Q)<0$. On the contrary, suppose that $\operatorname{rot}(Q) \geq 0$. If $\gamma^{-}<\gamma^{+}$, then $I(Q)>\gamma^{-}$, contrary to (2.3). Consider the case $\gamma^{-}=\gamma^{+}$. Fix a constant $0<\beta<\frac{1}{2} \sqrt{2 \alpha_{\varepsilon}}(|b-\xi|-2 \varepsilon)$. We can choose $T>1$ such that $Q([T, \infty)) \subset B_{\varepsilon}(b)$ and moreover,

$$
I_{-\infty}^{T}(Q)>\gamma^{-}-\beta \text {. }
$$

Since $q_{m}$ goes to $Q$ almost uniformly on $\mathbb{R}$, there is $m_{1} \in \mathbb{N}$ such that for all $m \geq m_{1}, \operatorname{rot}\left(\left.q_{m}\right|_{(-\infty, T]}\right)=\operatorname{rot}(Q)$, and hence $\operatorname{rot}\left(\left.q_{m}\right|_{[T, \infty)}\right)<-\operatorname{rot}(Q) \leq 0$. From Lemma 1.2 it may be concluded that

$$
I_{T}^{\infty}\left(q_{m}\right) \geq \sqrt{2 \alpha_{\varepsilon}}(|b-\xi|-2 \varepsilon)
$$

for $m \geq m_{1}$. Finally, by Remark 2.2, there exists $m_{2} \in \mathbb{N}$ such that for all $m \geq m_{2}$,

$$
I_{-\infty}^{T}\left(q_{m}\right)>I_{-\infty}^{T}(Q)-\frac{1}{4} \sqrt{2 \alpha_{\varepsilon}}(|b-\xi|-2 \varepsilon)
$$


By the above, for $m \geq \max \left\{m_{1}, m_{2}\right\}$ we obtain

$$
\begin{aligned}
I\left(q_{m}\right) & >I_{-\infty}^{T}(Q)+\frac{3}{4} \sqrt{2 \alpha_{\varepsilon}}(|b-\xi|-2 \varepsilon) \\
& >\gamma^{-}+\frac{1}{4} \sqrt{2 \alpha_{\varepsilon}}(|b-\xi|-2 \varepsilon),
\end{aligned}
$$

contrary to (2.2). Thus $Q \in \Gamma^{-}$, and, in consequence, $I(Q)=\gamma^{-}$.

Lemma 2.5. Assume that $V$ satisfies $(V 1)-(V 5)$ and $Z \in \mathcal{F}$. If $\left\{q_{m}\right\}_{m \in \mathbb{N}} \subset Z$ is a minimizing sequence of $I$ in $Z$ and $q_{0}$ is its weak limit in $E$, then $q_{0}$ is a connecting orbit of (1.1).

Proof. By assumption,

$$
\lim _{m \rightarrow \infty} I\left(q_{m}\right)=\inf \{I(q): q \in Z\} \equiv z .
$$

From Remark 2.2 and Corollary 1.3 it follows that $I\left(q_{0}\right) \leq z$ and $q_{0}( \pm \infty) \in$ $\mathcal{M}$. From Lemma 1.4 we deduce that $q_{0}(t) \neq \xi$ for all $t \in \mathbb{R}$. It is sufficient to prove that

$$
I\left(q_{0}\right)=\inf \left\{I(q): q \in \Lambda, q( \pm \infty)=q_{0}( \pm \infty) \wedge \operatorname{rot}(q)=\operatorname{rot}\left(q_{0}\right)\right\} .
$$

The rest of the proof runs as that of [6, Lemma 2.12] (see also [7, Lemma 4.3 and Lemma 4.5]). Therefore we omit it.

Proof of Theorem 1.6. As in the proof of Theorem 2.4, we assume that (2.1) holds.

Let $\left\{p_{m}\right\}_{m \in \mathbb{N}} \subset \Gamma^{+}$be a minimizing sequence of $I$ in $\Gamma^{+}$, i.e.,

$$
\lim _{m \rightarrow \infty} I\left(p_{m}\right)=\gamma^{+}
$$

Fix $0<\varepsilon \leq R$. Without restriction of generality, we can assume that for each $m \in \mathbb{N}$ there exists $\sigma_{m} \in[0,1)$ such that $p_{m}\left(\sigma_{m}\right) \in \partial B_{\varepsilon}(a)$ and if $t<\sigma_{m}$, then $\left|p_{m}(t)-a\right|<\varepsilon$. From Proposition 2.1 we deduce that there is $P \in E$ such that going to a subsequence if necessary $p_{m}$ goes to $P$ weakly in $E$ and strongly in $L_{\text {loc }}^{\infty}\left(\mathbb{R}, \mathbb{R}^{2}\right)$. From Lemma 1.4 it follows that $P \in \Lambda$. By Remark 2.2 we get

$$
I(P) \leq \gamma^{+},
$$

and by Corollary 1.3, $P( \pm \infty) \in \mathcal{M}$. Furthermore, $P((-\infty, 0]) \subset \operatorname{cl} B_{\varepsilon}(a)$, which implies $P(-\infty)=a$.

According to Lemma 2.5, $P$ is a connecting orbit of (1.1). Two cases are now possible: $P(\infty)=a$ or $P(\infty)=b$. If $P(\infty)=a$, then $P$ is a nontrivial homoclinic (to $a$ ) orbit of (1.1). We leave it to the reader to verify that $\operatorname{rot}(P)>0$. If $P(\infty)=b$ and $\operatorname{rot}(P) \geq 0$, then $P \neq Q$, where $Q$ is a heteroclinic solution of Theorem 2.4. If $P(\infty)=b$ and $\operatorname{rot}(P)<0$, then it may happen that $P=Q$. In this case, to receive the second connecting orbit of (1.1), we need the following lemma.

Lemma 2.6. If $P \in \Gamma^{-}$, then $\gamma^{+}=I(P)+\hat{\omega}_{b}^{n}$, where $n=-\operatorname{rot}(P)$. 
The proof of Lemma 2.6 is similar to that of [7, Lemma 4.4] for $V=$ $V(q)$. Therefore we omit it.

Assume that $P \in \Gamma^{-}$. Take $\left\{u_{m}\right\}_{m \in \mathbb{N}} \subset \hat{\Omega}_{b}^{n}$ such that

$$
\lim _{m \rightarrow \infty} I\left(u_{m}\right)=\hat{\omega}_{b}^{n} \text {. }
$$

There is no loss of generality in assuming that there is $\mu_{m} \in[0,1)$ such that $u_{m}\left(\mu_{m}\right) \in \partial B_{\varepsilon}(b)$ and if $t<\mu_{m}$, then $\left|u_{m}(t)-b\right|<\varepsilon$. Analysis similar to that for the sequence $\left\{p_{m}\right\}_{m \in \mathbb{N}}$ shows that there is $u \in \Lambda$ such that along a subsequence $u_{m}$ goes to $u$ weakly in $E$ and strongly in $L_{\text {loc }}^{\infty}\left(\mathbb{R}, \mathbb{R}^{2}\right)$. Moreover, $u$ is a connecting orbit of (1.1) such that $I(u) \leq \hat{\omega}_{b}^{n}, u((-\infty, 0]) \subset \operatorname{cl} B_{\varepsilon}(b)$ and $u(-\infty)=b$. If $u(\infty)=b$, then $u$ is a nontrivial homoclinic (to $b$ ) orbit of (1.1). The reader may check that $\operatorname{rot}(u)>0$. Consider now the case $u(\infty)=$ $a$. Let us remark that $\operatorname{rot}(u) \neq n$.

On the contrary, suppose that $\operatorname{rot}(u)=n$. By Lemma 2.3, there is $0<\delta \leq R$ such that

$$
\int_{T}^{T+1}\left(\frac{1}{2}|x-y|^{2}-V\left(t, l_{x, y}(t)\right)\right) d t<\frac{\eta}{2}
$$

for all $x, y \in B_{\delta}(a)$ and $T \in \mathbb{R}$. Choose $T>1$ such that $u([T, \infty)) \subset B_{\delta}(a)$. Since $u_{m}$ goes to $u$ almost uniformly on $\mathbb{R}$, there is $m_{0} \in \mathbb{N}$ such that for all $m \geq m_{0}$ we have $u_{m}([T, T+1]) \subset B_{\delta}(a)$ and $\operatorname{rot}\left(\left.u_{m}\right|_{(-\infty, T]}\right)=\operatorname{rot}(u)$. Let $v_{m}$ be given by

$$
v_{m}(t)= \begin{cases}a & \text { if } t \leq T, \\ (T+1-t) a+(t-T) u_{m}(T+1) & \text { if } t \in[T, T+1], \\ u_{m}(t) & \text { if } t \geq T+1,\end{cases}
$$

where $m \geq m_{0}$. Then $v_{m}(-\infty)=a, v_{m}(\infty)=b$ and

$$
\operatorname{rot}\left(v_{m}\right)=\operatorname{rot}\left(\left.u_{m}\right|_{[T+1, \infty)}\right)=\operatorname{rot}\left(u_{m}\right)-\operatorname{rot}\left(\left.u_{m}\right|_{(-\infty, T]}\right) \geq n-\operatorname{rot}(u)=0,
$$

and hence $v_{m} \in \Gamma^{+}$. Moreover,

$$
I\left(v_{m}\right) \leq \frac{\eta}{2}+I_{T+1}^{\infty}\left(u_{m}\right) \leq \frac{\eta}{2}+I\left(u_{m}\right) .
$$

Thus $\gamma^{+} \leq \frac{\eta}{2}+\hat{\omega}_{b}^{n}$, and consequently, $\gamma^{+} \leq \hat{\omega}_{b}^{n}$, contrary to Lemma 2.6.

Set $\bar{u}(t)=u(-t)$ for all $t \in \mathbb{R}$. As $\operatorname{rot}(u) \neq n$ we have $\operatorname{rot}(\bar{u}) \neq-n$, and so $\bar{u} \neq Q$. In consequence, even if $V$ is time reversible, the Newtonian system (1.1) possesses two geometrically distinct connecting orbits.

\section{Acknowledgment}

This research was supported by Grant of National Science Centre (Poland) no. 2011/03/B/ST1/04533.

\section{References}

[1] M. J. Borges, Heteroclinic and homoclinic solutions for a singular Hamiltonian system. European J. Appl. Math. 17 (2006), 1-32. 
[2] P. Caldiroli and L. Jeanjean, Homoclinics and heteroclinics for a class of conservative singular Hamiltonian systems. J. Differential Equations 136 (1997), $76-114$.

[3] W. B. Gordon, Conservative dynamical systems involving strong forces. Trans. Amer. Math. Soc. 204 (1975), 113-135.

[4] M. Izydorek and J. Janczewska, Homoclinic solutions for a class of the second order Hamiltonian systems. J. Differential Equations 219 (2005), 375-389.

[5] M. Izydorek and J. Janczewska, Heteroclinic solutions for a class of the second order Hamiltonian systems. J. Differential Equations 238 (2007), 381-393.

[6] M. Izydorek and J. Janczewska, The shadowing chain lemma for singular Hamiltonian systems involving strong forces. Cent. Eur. J. Math. 10 (2012), 1928-1939.

[7] J. Janczewska, The existence and multiplicity of heteroclinic and homoclinic orbits for a class of singular Hamiltonian systems in $\mathbb{R}^{2}$. Boll. Unione Mat. Ital. (9) 3 (2010), 471-491.

[8] P. H. Rabinowitz, Periodic and heteroclinic orbits for a periodic Hamiltonian system. Ann. Inst. H. Poincaré Anal. Non Linéaire 6 (1989), 331-346.

[9] P. H. Rabinowitz, Homoclinics for a singular Hamiltonian system. In: Geometric Analysis and the Calculus of Variations, Int. Press, Cambridge, MA, 1996, $267-296$.

[10] K. Tanaka, Homoclinic orbits for a singular second order Hamiltonian system. Ann. Inst. H. Poincaré Anal. Non Linéaire 7 (1990), 427-438.

Marek Izydorek

Faculty of Applied Physics and Mathematics

Gdańsk University of Technology

G. Narutowicza 11/12

80-233 Gdańsk

Poland

e-mail: izydorek@mif.pg.gda.pl

Joanna Janczewska

Faculty of Applied Physics and Mathematics

Gdańsk University of Technology

G. Narutowicza 11/12

80-233 Gdańsk

Poland

e-mail: janczewska@mif.pg.gda.pl

Open Access This article is distributed under the terms of the Creative Commons Attribution License which permits any use, distribution, and reproduction in any medium, provided the original author(s) and the source are credited. 